\title{
Perforación de la uretra proximal durante TVT-O, en paciente con desviación uretral secundaria a cirugia de Burch fallida
}

\author{
Paolo Ricci Arriola*, Vicente Solà Dalenz, Jack Pardo Schanz \\ Unidad de Uroginecología y Cirugía Vaginal de Clínica Las Condes. Santiago. Chile.
}

\begin{abstract}
Resumen
Mujer de 59 años con antecedentes de Burch laparoscópico realizado durante el 2003 en otro recinto hospitalario. La paciente acude a nuestro centro el año 2006 por persistencia de una incontinencia urinaria, que en el estudio urodinámico mostró una incontinencia de esfuerzo tipo II y una vejiga inestable motora sin evidencia de obstrucción. Durante la realización de un TVT-O (Tensión-free Vaginal Tape Obturador in-out route: cinta libre de tensión vía obturador desde adentro hacia afuera), se evidenció salida de sangre por la sonda Foley después del paso de la aguja por el agujero obturador derecho. La cistoscopia demostró una desviación significativa de la uretra hacia la derecha y el paso de la cinta a través de la uretra proximal. La cinta se pasó nuevamente tomando las precauciones necesarias. Dos días después de la operación se retiró la sonda y se comprobó la ausencia de incontinencia de esfuerzo. Los síntomas de urgencia miccional remitieron con tratamiento médico (Tolteridina $2 \mathrm{mg}$ /día). Creemos que la desviación uretral causada por un Burch laparoscópico técnicamente deficiente fue la causa de la perforación uretral ocurrida durante el TVT-O.
\end{abstract}

Palabras clave: TVT-O. Complicación de TVT-O. Perforación de uretra.

\section{Proximal urethra portion perforation during TVT-O in a patient with urethral deviation secondary to previous failed Burch surgery \\ Abstract}

A 59 years old woman with laparoscopic Burch made during 2003 in another hospital. During 2006, the patient is evaluating in our center for a persistent urinary incontinence, the urodynamic study demonstrated stress urinary incontinence type II and overactive bladder without obstruction evidences. A TVT-O (tension-free vaginal tape obturador in-out route) was made, nevertheless when the right branch was passed blood was observed in the Foley catheter. A cystoscopy showed an important deviation of urethra and the tape through urethra in the proximal portion. The right branch went again inserted taking the necessary precautions. Two days after surgery the Foley catheter was removed and the stress urinary incontinence symptoms disappeared. The urge incontinence symptoms disappeared with the pharmacalogical treatment (Tolterodine $2 \mathrm{mg} /$ day). We believe that the urethral deviation caused by a technically deficient laparoscopic Burch was the reason for the urethral perforation during the TVT-O.

Keywords: TVT-O. Complication of TVT-O Urethra perforation.

$\mathrm{L}$ as cirugías de cinta bajo la uretra media han probado su eficacia en la corrección quirúrgica de la incontinencia de orina de esfuerzo ${ }^{1,2}$. La primera de ellas fue el TVT (Tension-free Vaginal Tape), presentado en 1996 por Ulmsten ${ }^{3}$. Más tarde se logró simplificar la técnica quirúrgica, mediante la salida de la cinta a través de los agujeros obturadores, con la técnica de TOT (Transobturator tape out- in route) ${ }^{4}$ y TVT-O (tension-free vaginal tape obturator in-out route $)^{5,6}$. De esta manera se disminuye el tiempo operatorio y la posibilidad de perforación de la vejiga y de la vía urinaria ${ }^{7}$.

A continuación presentamos un caso de perforación y paso de la cinta de TVT-O por la uretra, en una paciente con desviación anatómica de esta, secundaria a una cirugía de Burch fallida. 


\section{CASO CLÍnICo}

Mujer de 59 años, multípara de 4 partos vaginales, obesa, con terapia de restitución hormonal. Antecedente de operación de Burch, realizada durante el año 2003 mediante laparoscopia en otro recinto hospitalario. En esa ocasión se indicó la cirugía por una incontinencia de orina diagnosticada por clínica, sin estudio de urodinamia. Relata fracaso de la cirugía evidenciado desde el postoperatorio inmediato.

En el momento de la consulta en nuestra unidad, en mayo del 2006, se detectan clínicamente síntomas de incontinencia de orina frente a mínimos esfuerzos y componentes de urgencia. Se descarta la presencia de prolapso y se comprueba hipermovilidad uretral mediante un $\mathrm{Q}$-Tip-Test (quantification Tip Test). El estudio urodinámico demostró incontinencia de orina de esfuerzo tipo II y una vejiga inestable, sin elementos de obstrucción.

Se realizó un TVT-O con técnica clásica ${ }^{5}$, sin embargo al pasar la aguja por el lado derecho, se observó salida de orina teñida con sangre por la sonda uretral. Por este motivo se realizó una cistoscopia de revisión, por medio de la cual se evidenció una desviación de la uretra hacia la derecha y la presencia de la cinta dentro de la porción más proximal (cercana a la vejiga) (Figs. 1 y 2). Se retiró la cinta bajo visión de cistoscopia, comprobando la retracción de la perforación en la pared uretral, y ausencia de sangrado activo.

Se colocó nuevamente la cinta repitiendo la técnica, con la precaución de vaciar completamente la vejiga y alejarse lo más posible de la uretra. Se dejó una sonda de silicona por 48 horas. Los síntomas de incontinencia de orina de esfuerzo desaparecieron y se indicó Tolterodina $2 \mathrm{mg}$ al día posteriormente para los sintomas de vejiga inestable. En el primer control postoperatorio se comprobó la presencia de la cinta en la porción sub-medio-uretral. La paciente a la fecha completó un año de controles y permanece asintomática.

\section{COMENTARIOS}

Una técnica quirúrgica deficiente durante el Burch laparoscópico parece ser la causa de la desviación uretral y del fracaso en la resolución de la incontinencia evidenciado desde el postoperatorio inmediato en este caso.

Esta desviación anatómica fue corroborada mediante la cistoscopia. A la inspección se logró demostrar una
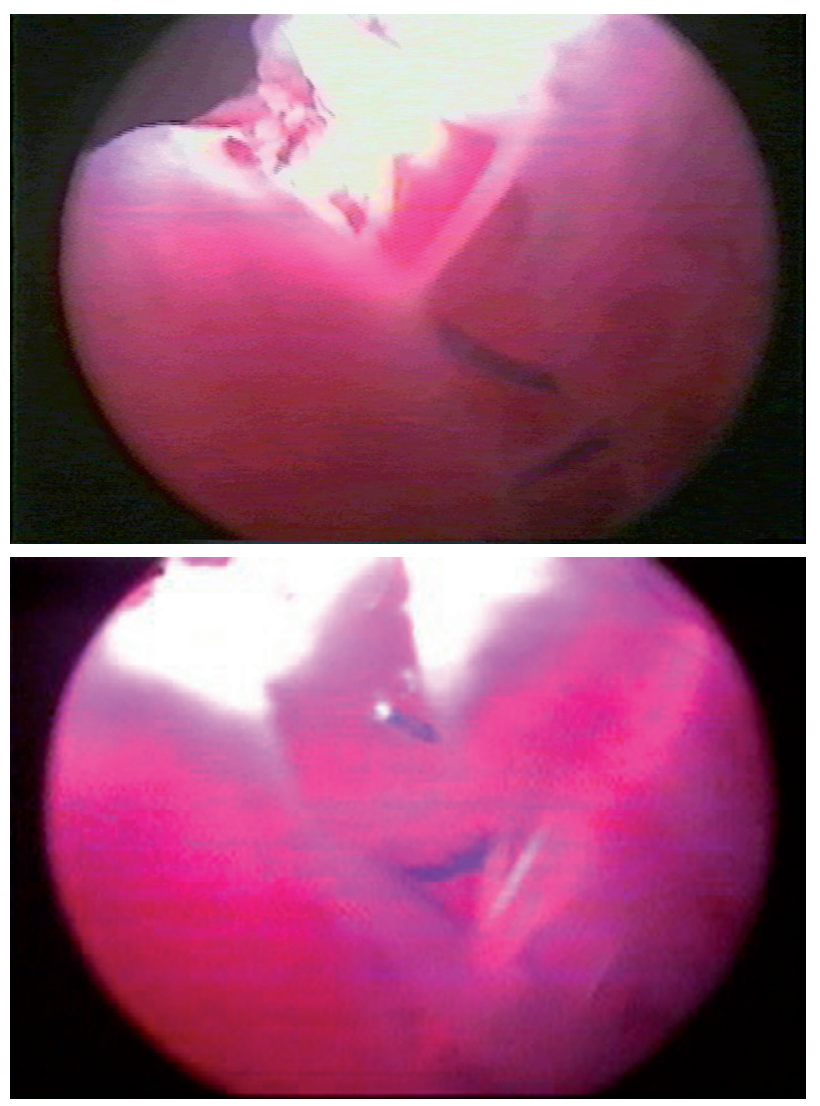

FIGURAS 1 y 2. Visión de la cinta en el interior de la uretra proximal.

desviación hacia la derecha del eje medio uretral, que muy probablemente se debió a la tracción asimétrica de los puntos de la operación de Burch.

En la planificación preoperatorio, se tuvo en cuenta la posibilidad de realizar un nuevo Burch, sin embargo significaba realizar una nueva cirugía en una zona con probable fibrosis secundaria al antecedente quirúrgico. Además la paciente solicitaba una cirugía con mínima invasión. Por estas razones se decidió realizar una técnica de cinta submedio uretral con salida por el agujero obturador. Técnica quirúrgica que a la fecha actual, hemos logrado exitosamente en 230 pacientes. Sin embargo, y aún cuando se tomaron todas las precauciones que hasta ese momento se creía eran suficientes, el alejamiento de la aguja no fue suficiente y perforamos la uretra.

Sin embargo, a pesar de que la perforación uretral es una de las complicaciones temidas al momento de aplicar cintas sub-medio-uretrales, la solución no fue compleja. Se retiró la cinta y se colocó nuevamente, con la precaución de alejarse más aún de la uretra desviada. No se realizó otra inter- 
vención ni se tomó otra medida adicional, ya que la cirugía logró revertir la incontinencia de orina de esfuerzo, y en conjunto con la terapia medicamentosa se revertieron completamente los sintomas que motivaron la consulta.

En experiencias publicadas previamente con esta técnica, no habíamos reportado complicaciones de perforación de vejiga ni de vía urinariaa ${ }^{5,7}$. Esta técnica es segura y efectiva ${ }^{8,9}$, con una baja posibilidad de dañar la vía urinaria y en este caso fue debido a la alteración anatómica de la uretra, muy posiblemente como resultado de un Burch asimétrico y mal logrado.

Una publicación de Abdel-Fattah y colaboradores $^{10}$, durante el 2006, evidenció que las únicas 2 perforaciones uretrales ocurridas durante una serie de 390 operaciones de TOT, ocurrieron en pacientes con antecedente de cirugía previa. El presente caso parece aportar a la idea, de la necesidad de realizar una cistoscopia en las operaciones de TOT o TVT-O realizadas en paciente previamente intervenidas, por existir un pequeño riesgo de perforación uretral.

\section{CONCLUSIÓN}

Aún cuando el TVT-O es una técnica con menor probabilidad de perforación de la vejiga y de la uretra, al compararla con su antecesor el TVT. Una desviación anatómica de la uretra puede ser la causa de esta complicación.

Cuando se realiza un TOT o un TVT-O en mujeres con antecedente de alguna cirugía que podría haber afectado a la uretra, un estudio previo de vía urinaria podría permitir identificar posibles alteraciones anatómicas, para disminuir la probabilidad de perforación.

\section{REFERENCIAS}

1. Latthe PM, Foon R, Toozs-Hobson P. Transobturator and retropubic tape procedures in stress urinary incontinence: a systematic review and meta-analysis of effectiveness and complications. BJOG. 2007;114(5):522-531.

2. Zullo MA, Plotti F, Calcagno M, Marullo E, Palaia I, Bellati F, Basile S, Muzii L, Angioli R, Panici PB. One-year follow-up of tension-free vaginal tape (TVT) and trans-obturator suburethral tape from inside to outside (TVT-O) for surgical tratment of female stress urinary incontinence: a prospective randomised trial. Eur Urol. 2007;51(5):1376-1382; discussion 1383-1384.

3. Ulmsten U, Henriksson L, Johnson P, Varhos G. An ambulatory surgical procedure under local anesthesia for treatment of female urinary incontinence. Int Urogynecol $\mathrm{J}$ Pelvic Floor Dysfunct. 1996;7(2):81-85.

4. Pardo Schanz J, Ricci Arriola P, Sola Dalenz V, Tacla Fernández X. Trans-obturator-tape (TOT) for the surgical repair of stress urinary incontinence: our experience. Arch Esp Urol. 2006;59 (3):225-232.

5. Sola Dalenz V, Pardo Schanz J, Ricci Arriola P, Guiloff Fische E, Chiang Miranda H. Cirugía mínimamente invasiva en la incontinencia urinaria femenina: TVT-O. Actas Urol Esp. 2006; 30(1): 61-66.

6. De Leval J, Waltregny D. New surgical technique for treatment of stress urinary incontinence TVT-Obturator: new developments and results. Surg Technol Int. 2005;14:212-221.

7. Sola V, Pardo J, Ricci P, Guiloff E, Chiang H. TVT versus TVT-O for Minimally Invasive Surgical Correction of Stress Urinary Incontinence. Int Braz J Urol. 2007;33(2):246-253; discussion 254.

8. Lim J, Cornish A, Carey MP. Clinical and quality-of-life outcomes in women treated by the TVT-O procedure. BJOG. 2006; 113(11):1315-1320.

9. Lim JL, Quinlan DJ. Safety of a new transobturator suburethral synthetic sling (TVT-O) procedure during the training phase. J Obstet Gynaecol Can. 2006;28(3):214-217.

10. Abdel-Fattah M, Ramsay I, Pringle S. Lower urinary tract injuries after transobturator tape insertion by different routes: a large retrospective study. BJOG 2006, 113(12):1377-1381.

Correspondencia autor: Dr. Paolo Ricci Arriola Unidad de Uroginecología y Cirugía Vaginal

Clínica Las Condes. Lo Fontecilla 441, Las Condes,

Santiago, Chile. Tel.: (56) 22104610

E-mail autor: pricci@clinicalascondes.cl / pricci@vtr.net

Información artículo: Nota clínica

Trabajo recibido: junio 2007

Trabajo aceptado: enero 2009 